\title{
LA TRANSFERENCIA DEL MANDO EN LAS PEQUEÑAS Y MEDIANAS EMPRESAS FAMILIARES
}

\section{THE TRANSFER OF COMMAND IN SMALL AND MEDIUM FAMILY COMPANIES}

\author{
Mariela Adriana Rodríguez Ocaña*, Erik Haidar Torres**, \\ Abel Pérez Sánchez***
}

\begin{abstract}
*Doctorante en Administración. Universidad Autónoma de Guadalajara, Campus Tabasco. ORCID: https://orcid.org/0000-0002-3110-7439.

**Doctor en Administración. Email: erikhaidar@gmail.com. ORCID: https://orcid.org/0000-0002-58564736.

***Doctor en Administración. Universidad Autónoma de Guadalajara, Campus Tabasco. Email: aperezs@uagtabasco.edu.mx. ORCID: https://orcid.org/0000-0002-0088-1122.
\end{abstract}

Dirección para recibir correspondencia: marielaadrianarodriguezocana@gmail.com 
LA TRANSFERENCIA DEL MANDO EN LAS PEQUEÑAS Y MEDIANAS EMPRESAS FAMILIARES

\section{RESUMEN}

OBJETIVO: Determinar los factores que intervienen en la transferencia del mando en la gestión en las PYMES familiares determinada por un proceso de toma de decisión donde confluyen intereses contradictorios; los de la familia e individuos, los propios de la organización y las demandas que hace el entorno donde la PYME desarrolla su actividad.

MATERIAL Y METODO: Se realiza un estudio observacional, bajo un enfoque cuantitativo, de corte transversal y exploratorio en su primera etapa en PYMES del Estado de Tabasco, México. Con base en la revisión de la literatura que aborda las premisas de decisión y la sucesión de empresas, se realizó la construcción de un modelo teórico e instrumento de investigación centrado en las premisas para la decisión de Luhmann: personal, comunicación interna y programas.

RESULTADOS: A través de la perspectiva de modelación de los sistemas sociales, se observan los mecanismos en que pueden ser afrontadas las decisiones para la transferencia de mando que pueden garantizar la continuidad organizacional, así como determinar cuáles serían las premisas para la decisión en el proceso de transferencia de mando en las PYMES Familiares.

CONCLUSIONES: Mediante resultados de análisis factorial exploratorio de las variables que conducen a indicadores objetivos y representan en sí la información relevante que da respuesta a las preguntas de investigación. Cada variable deviene de estudios teóricos y empíricos referidos en la literatura sobre la toma de decisión para la sucesión en las organizaciones.

PALABRAS CLAVE: Decisión. Sucesión. Racionalidad de la información.

\section{ABSTRACT}

OBJECTIVE: To determine the involved factors in the transfer of command of the management of family SMEs determined by a decision-making process where contradictory interests converge; those of the family and individuals, those of the organization itself and the demands made by the environment where the SME develops its activity. 
LA TRANSFERENCIA DEL MANDO EN LAS PEQUEÑAS Y MEDIANAS EMPRESAS FAMILIARES

MATERIAL AND METHOD: An observational study in SMEs of the State of Tabasco, Mexico was carried out under a quantitative approach, which was cross-section and exploratory in its first stage. Based on the review of the literature that addresses the Decision Premises and the Succession of Companies, the construction of a theoretical model and research instrument focused on the premises for Luhmann's decision: personnel, internal communication and programs was also built.

RESULTS: Through the perspective of modeling Social Systems, we observe the mechanisms in which the decisions for the transfer of command can be faced, mechanisms that can guarantee organizational continuity, as well as the premises for the decision in the process of transfer of command in the Family SMEs.

CONCLUSIONS: Through exploratory factor analysis results of the variables that lead to objective indicators, variables which represent the relevant information that answers the research questions. Each variable mentioned on the literature about decision making for succession in organizations comes from theoretical and empirical studies

KEY WORDS: Decision. Succession. Rationality of information.

\section{INTRODUCCIÓN}

Las perspectivas epistemológicas para el estudio de las decisiones organizacionales se pueden dividir en dos grupos: racionalistas y constructivistas. Los estudios racionalistas tratan de explicarlas en términos instrumentales y causales, mientras que los estudios constructivistas lo hacen desde una perspectiva sistémica; es decir, como una construcción organizacional (Espinosa, 2016).

Bajo la perspectiva de los sistemas sociales de Niklas Luhmann, las decisiones organizacionales se conciben como operaciones de comunicación autoproducidas en respuesta al entorno donde la organización realiza su actividad y se circunscriben a premisas de decisión en los ámbitos personal, jerárquico y de los programas (Espinosa, 2016). 
LA TRANSFERENCIA DEL MANDO EN LAS PEQUEÑAS Y MEDIANAS EMPRESAS FAMILIARES

Las premisas de decisión no son más que presupuestos de información que hacen que la información para las decisiones organizacionales sea manejable; se reduce la complejidad asociada a los procesos de decisión, considerando la experiencia adquirida por la organización en decisiones anteriores y evitando que cada vez se tenga que evaluar la complejidad de cada situación. Son redundancias que reducen la carga de información a un formato soportable (Luhmann, 2010, p. 66).

La complejidad asociada a las decisiones organizacionales no solo radica en los criterios de productividad o los objetivos organizacionales que necesariamente deben observarse, también se relaciona con la permanencia de la organización y su adaptación al entorno, a esta complejidad se suma lo que Herbert A. Simon denominó racionalidad limitada en la toma de decisiones.

Los Modelos típicos de decisión asumen que todas las posibles alternativas son conocidas, que todas las consecuencias relativas a cada alternativa también son conocidas y que los beneficios asociados a cada una de ellas, también pueden conocerse con antelación. La racionalidad consiste en la optimización del beneficio; elegir la alternativa de mayor utilidad. En la práctica, la información que poseen los que toman decisiones con relación a las posibles alternativas 0 cursos de acción, las consecuencias y los beneficios asociados a éstas, es escasa e incierta; la racionalidad está limitada a las condiciones de escasez e incertidumbre de la información para la decisión (Simon, 1979).

Desde la teoría de la decisión de Herbert A. Simon, las organizaciones toman decisiones orientadas al cumplimiento de sus propios objetivos, de manera adaptativa, en un contexto que supera por mucho su capacidad de procesamiento de información. Propone en su teoría que las organizaciones deben constantemente estar pendientes de los aspectos del entono que reclaman de su atención, para diseñar respuestas adaptativas a tales requerimientos y elegir las acciones a realizar.

Las experiencias previas y la experimentación constituyen dos de los mecanismos básicos para la selección de las mejores alternativas como respuesta adaptativa; la información registrada del éxito de las soluciones previas son la base para las decisiones futuras. Esto es más evidente cuando el problema a resolver es similar a uno que ya fue resuelto con éxito en el pasado. De esta manera, se minimiza el mecanismo de prueba-error (Simon, 1962). 
LA TRANSFERENCIA DEL MANDO EN LAS PEQUEÑAS Y MEDIANAS EMPRESAS FAMILIARES

Las premisas enfocan la comunicación... y eso hace probable que las decisiones futuras sean observadas en referencia a premisas dadas en lugar de desarrollar cada vez, nuevamente, toda la complejidad de las situaciones (Luhmann, 2010, p. 263). Además, establecen criterios que delimitan las decisiones, no sólo en lo jerárquico (vías de comunicación), también los rasgos personales de los miembros de la organización que modulan la decisión y la manera en que se toman las decisiones organizacionales.

Para tomar decisiones efectivas se hace necesario valorar la información relevante y pertinente a la organización, sus integrantes y del entorno. A esta información relevante para la decisión es a lo que denominamos premisas para la decisión.

De lo anterior, se esboza la pregunta de investigación: ¿Cuáles serían las premisas para la decisión en el proceso de transferencia de mando en las PYMES familiares?

Para abordar la problemática anteriormente definida, se procedió a elaborar un modelo teórico basado en los estudios previos sobre las decisiones para el ascenso, referidos en la literatura y la modelización de sistemas (Eriksson, 1997).

\section{La modelación de los sistemas complejos}

Se toma como unidad de estudio la decisión para el ascenso. Se considera que en las decisiones para los ascensos se observan las propiedades del fenómeno que se pretende investigar y para lo cual el modelo es pertinente; las premisas para la decisión en el proceso de transferencia de mando.

En este sentido, se investigan las propiedades emergentes (Eriksson, 1997) como atributos en las decisiones para los ascensos dentro de la estructura de mando de las PYMES. Estas propiedades se manifiestan a modo de información relevante para la decisión o premisas (Luhmann, 2010).

Los estudios previos sobre las decisiones para el ascenso, han sido abordados desde las vertientes: Racional (Danco, 1975; Simon, 1979), Recursos y Capacidades (Coleman, 2006), Del fundador (Hollander, 1988), Evolutivo (Barnes y Hershon, 1999) y De planificación (Domínguez, 2011; Schumacher, 2015). En la tabla 1, se muestra la información relevante o premisa que cada enfoque considera para la selección de la mejor alternativa en la decisión para el ascenso. 


\section{LA TRANSFERENCIA DEL MANDO EN LAS PEQUEÑAS Y MEDIANAS EMPRESAS FAMILIARES}

\section{Tabla 1}

Información relevante de los enfoques para el estudio de las decisiones para el ascenso

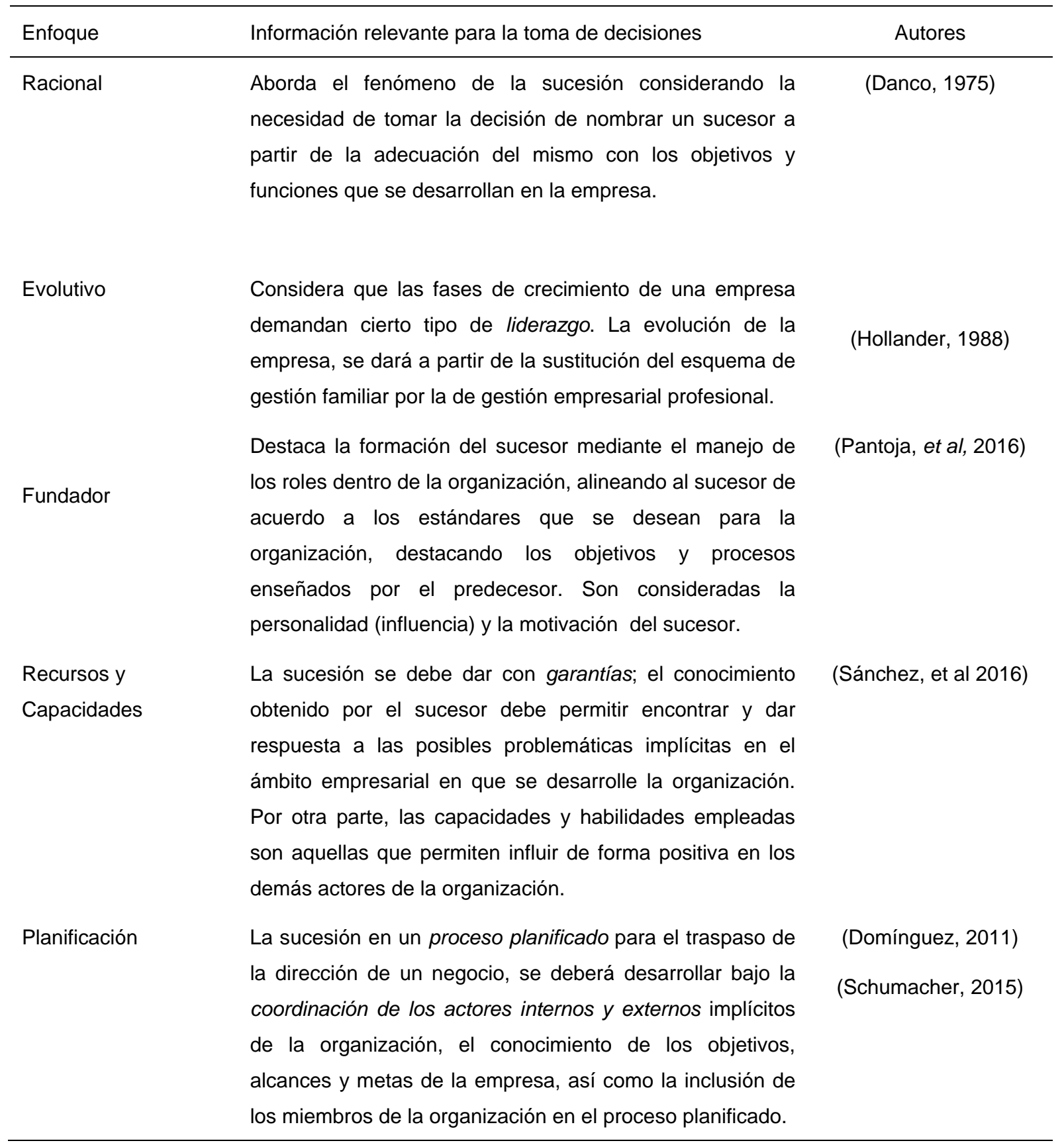

Nota: Se especifican los autores en cada fila. Se nombra a algunos de los autores relevantes sin pretender ser exhaustivos.

Fuente: Elaboración propia, basada en la revisión de la literatura relevante al tema. 


\section{LA TRANSFERENCIA DEL MANDO EN LAS PEQUEÑAS Y MEDIANAS EMPRESAS FAMILIARES}

Para garantizar la coherencia del modelo propuesto, basado en la teoría de sistemas de Luhmann, con los modelos para el estudio de las decisiones para la sucesión que se presentan en la tabla 1, se toman como componentes para agrupar las variables del modelo, los tres grandes tipos de premisas para la decisión de Luhmann (2010). En primer lugar, los programas de decisión; que refieren a cómo decidir correctamente; en segundo lugar, las vías de comunicación; que refieren a las competencias asociadas al nivel jerárquico para la toma de decisiones específicas; $y$, en tercer lugar, uso del personal; que refiere a las premisas asociadas a los rasgos del personal que modulan las decisiones individuales; es decir, sobre su reclutamiento y ubicación. De esta manera, se construye un modelo que incluye las variables relativas a cada enfoque, dando cuenta de la información relevante para las premisas de decisión en la sucesión (ver tabla 2).

\section{Tabla 2}

Variables con las premisas para la decisión en la sucesión

\begin{tabular}{|c|c|c|}
\hline $\begin{array}{l}\text { Premisas generales de } \\
\text { Luhmann }\end{array}$ & Dimensión & $\begin{array}{c}\text { Variable } \\
\text { (Información para la decisión) }\end{array}$ \\
\hline Uso del personal & $\begin{array}{l}\text { Enfoque } \\
\text { Racional }\end{array}$ & $\begin{array}{l}\text { Adecuación del sucesor: } \\
\text { (Adecuación del sucesor con los objetivos y funciones que se } \\
\text { desarrollan en la empresa). }\end{array}$ \\
\hline Uso del personal & $\begin{array}{l}\text { Enfoque de } \\
\text { Recursos y } \\
\text { Capacidades }\end{array}$ & $\begin{array}{l}\text { Garantías: } \\
\text { (El sucesor debe encontrar y dar respuesta a las posibles } \\
\text { problemáticas implícitas en el ámbito empresarial en que se } \\
\text { desarrolle la organización). } \\
\text { Capacidades de Influir: } \\
\text { (Capacidades y habilidades que permiten influir de forma positiva } \\
\text { en los demás actores de la organización). }\end{array}$ \\
\hline Programas de decisión & $\begin{array}{l}\text { Enfoque del } \\
\text { Fundador }\end{array}$ & $\begin{array}{l}\text { Formación previa: } \\
\text { (La formación del sucesor mediante el manejo de los roles dentro } \\
\text { de la organización, alineando al sucesor de acuerdo a los } \\
\text { estándares que se desean para la organización, destacando los } \\
\text { objetivos y procesos enseñados por el predecesor) }\end{array}$ \\
\hline & & $\begin{array}{l}\text { Influencia y Motivación: } \\
\text { (Son consideradas la personalidad y la motivación del sucesor) }\end{array}$ \\
\hline
\end{tabular}


LA TRANSFERENCIA DEL MANDO EN LAS PEQUEÑAS Y MEDIANAS EMPRESAS FAMILIARES

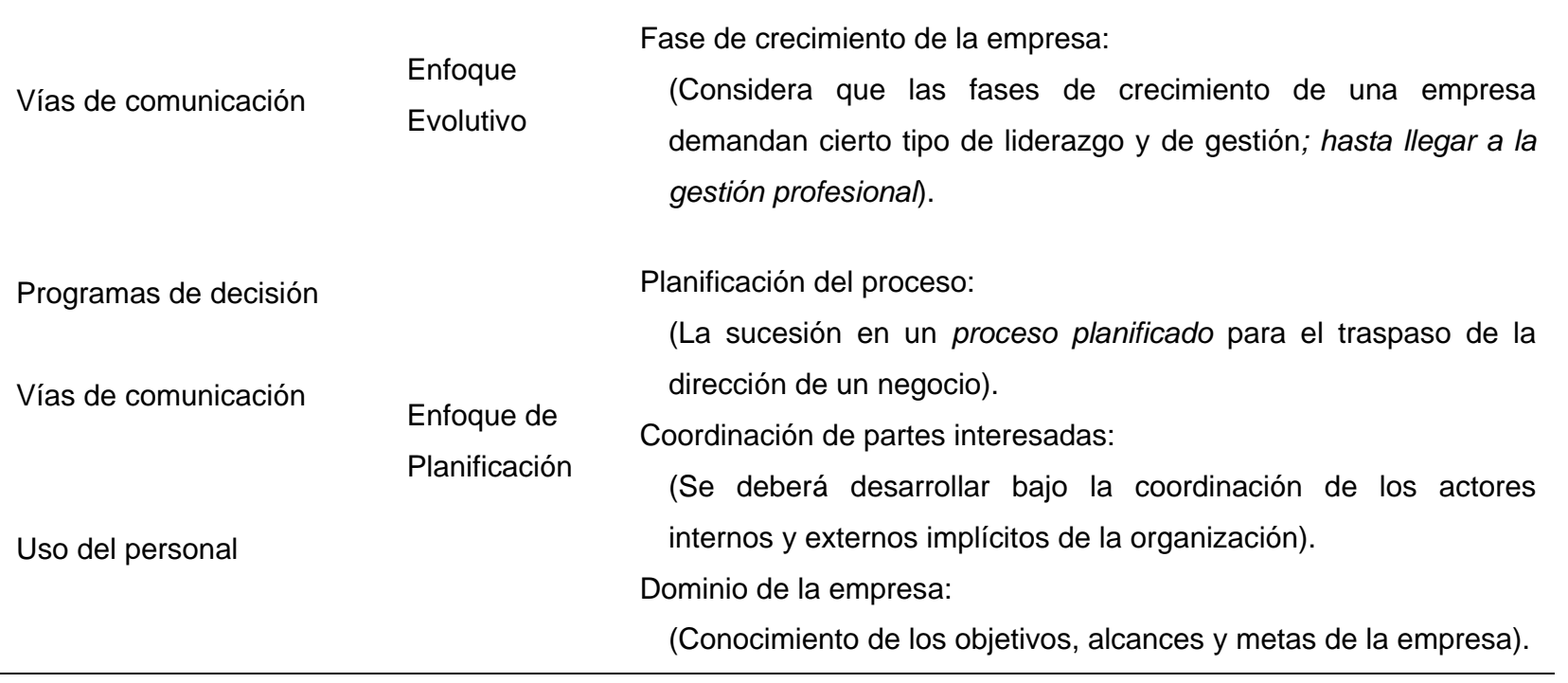

Fuente: Elaboración propia, basada en la revisión de la literatura relevante al tema.

En la figura 1, se presenta un esquema del modelo teórico. Se consideran como componentes a las Premisas para la Decisión de Luhmann: Programas de Decisión, Vías de Comunicación y Uso del Personal, además, se muestran las variables asociadas a cada uno de ellos y que son el aporte de las investigaciones previas sobre el tema desde perspectivas diversas. Se integran como hipótesis, lo relevante de los estudios teóricos realizados sobre el tema; los tres componentes de las premisas de decisión de Niklas Luhmann y las variables asociadas a cada uno desde los enfoques Racional (Danco, 1975), Recursos y Capacidades (Sánchez, et al 2016), del Fundador (Pantoja, et al, 2016), Evolutivo (Hollander, 1988) y de Planificación (Domínguez, 2011; Schumacher, 2015). 


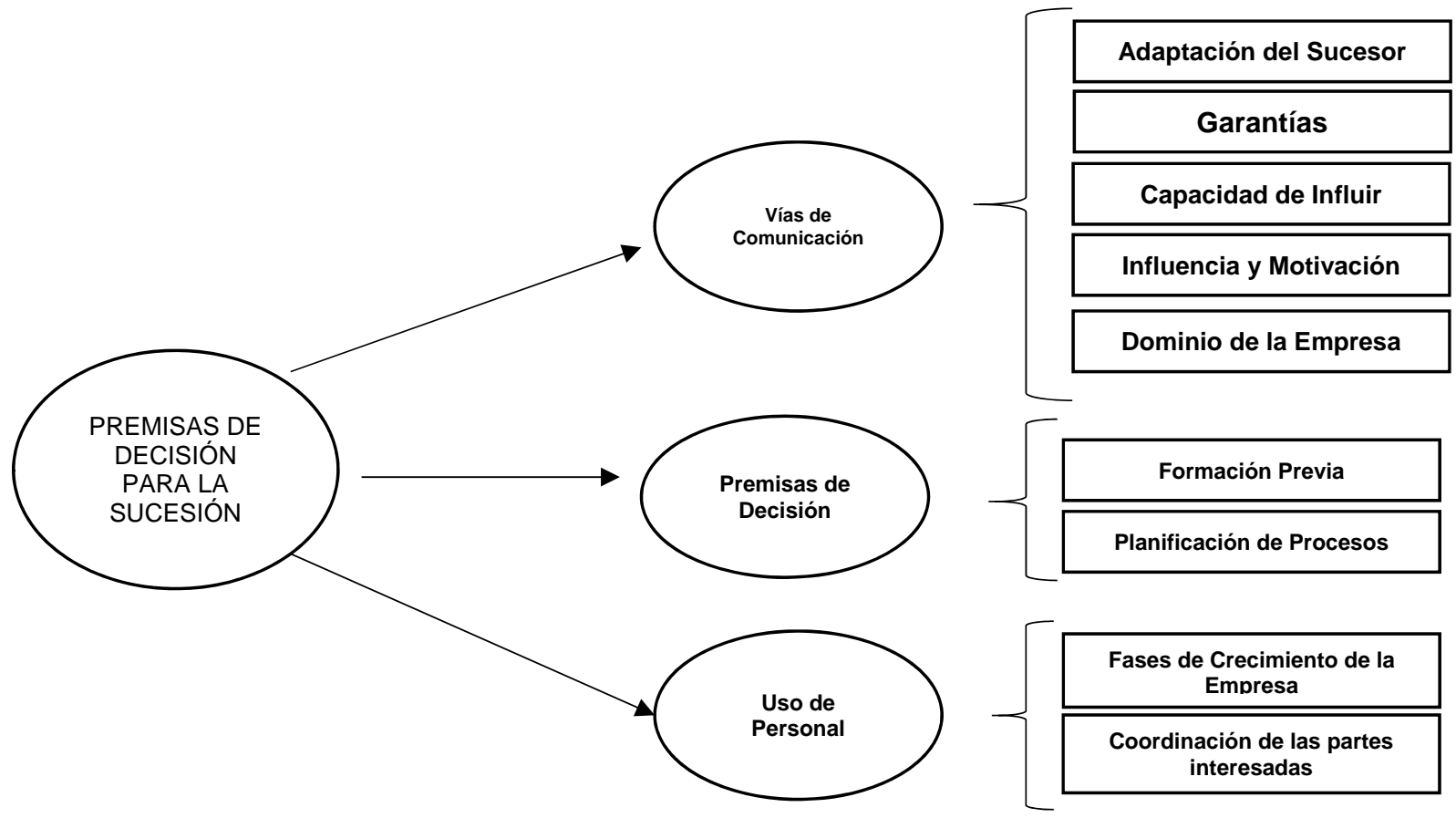

Figura 1. Modelo teórico de las premisas de decisión para la sucesión.

Fuente: Modelo elaborado desde la teoría de la decisión de Niklas Luhmann y Herbert A. Simon, así como de estudios previos desde los enfoques Racional (Danco, 1975), Recursos y Capacidades (Sánchez, et al 2016), del Fundador (Pantoja, et al, 2016), Evolutivo (Hollander, 1988) y de Planificación (Domínguez, 2011; Schumacher, 2015).

\section{MATERIAL Y MÉTODO}

Se realiza un estudio observacional, bajo un enfoque cuantitativo, de corte transversal y exploratorio, en PYMES del Estado de Tabasco, México.

Se construyó un cuestionario como instrumento para medir los indicadores de las variables del modelo. En la tabla 3, se muestran los componentes, las variables y los indicadores utilizados para la operacionalización del modelo teórico.

Se realizó un análisis factorial exploratoria, basada en el método de Componentes Principales, dada la naturaleza formativa del constructo. A nivel de las variables individuales, se realizaron Análisis Factorial Exploratorio (AFE) utilizando Máxima Verosimilitud, dada la naturaleza reflectiva de los indicadores en este nivel. 


\section{LA TRANSFERENCIA DEL MANDO EN LAS PEQUEÑAS Y MEDIANAS EMPRESAS FAMILIARES}

Para cada indicador, se diseñaron ítems acorde a su valor sustantivo. En todos los casos se consideraron las escalas desarrolladas con anterioridad y referidas en la tabla 3.

\section{Tabla 3}

Componentes, variables e indicadores para la operacionalización del modelo teórico

\begin{tabular}{|c|c|c|c|}
\hline Componentes & Variables & Indicadores & Autor (es) \\
\hline \multirow{4}{*}{ Uso del Personal } & Adaptación del sucesor & $\begin{array}{c}\text { Autoridad } \\
\text { Información } \\
\text { Integración }\end{array}$ & $\begin{array}{c}\text { (Hania, 2012) } \\
\text { (Schumacher, 2015) }\end{array}$ \\
\hline & $\begin{array}{l}\text { Garantías y capacidad } \\
\text { de influir }\end{array}$ & $\begin{array}{l}\text { Trabajo en Equipo } \\
\text { Habilidad y Talentos }\end{array}$ & $\begin{array}{c}\text { (Colquitt J. , 2011) } \\
\text { (Schumacher, 2015) }\end{array}$ \\
\hline & Influencia y motivación & Influencia & $\begin{array}{l}\text { (Hania, 2012) } \\
\text { (Dana, 2015) }\end{array}$ \\
\hline & Dominio de la empresa & Entorno & (Hania, 2012) \\
\hline \multirow{2}{*}{ Programas de Decisión } & Formación previa & Motivación Personal & $\begin{array}{l}\text { (Hania, 2012) } \\
\text { (Dana, 2015) }\end{array}$ \\
\hline & $\begin{array}{l}\text { Planificación de } \\
\text { procesos }\end{array}$ & Planeación & (Hania, 2012) \\
\hline \multirow{2}{*}{ Vías de Comunicación } & $\begin{array}{l}\text { Fases de crecimiento } \\
\text { de la empresa }\end{array}$ & $\begin{array}{l}\text { Liderazgo } \\
\text { Confiabilidad }\end{array}$ & $\begin{array}{l}\text { (Coffman, 2014) } \\
\text { (Goana, 2015) }\end{array}$ \\
\hline & $\begin{array}{l}\text { Coordinación de las } \\
\text { partes interesadas }\end{array}$ & Microambiente & (Hania, 2012) \\
\hline
\end{tabular}

Fuente: Basado en el modelo teórico y los autores citados en la tabla.

El cuestionario consta de 75 ítems y datos sociodemográficos. El formato de respuesta de las preguntas corresponde a una escala tipo Likert de 7 puntos, con los siguientes valores: 1= Totalmente en desacuerdo, 2= Muy en desacuerdo, 3= En desacuerdo, 4= Ni de acuerdo, ni en desacuerdo, 5= De acuerdo, 6= Muy de acuerdo y $7=$ Totalmente de acuerdo.

\section{Población y tamaño de muestra}

La población objeto de estudio se corresponde a la totalidad de PYMES que radican en el municipio del Centro, en Villahermosa, Tabasco, México. Dada la imposibilidad de contar con un padrón de PYMES, se optó por un muestreo por conglomerados, tomando a las cámaras y colegios como tales. Dentro de éstas, se intervinieron a los empleados de las PYMES que accedieron a brindar información para la investigación (ver tabla 4). 
LA TRANSFERENCIA DEL MANDO EN LAS PEQUEÑAS Y MEDIANAS EMPRESAS FAMILIARES

Tabla 4

Padrón cámaras y colegios

\begin{tabular}{lll}
\hline Padrón PYMES & No. de socios & $\mathrm{n}$ \\
\hline CMIC & 400 & 13 \\
CANACO & 52 & 48 \\
IMCP & 197 & 18 \\
CCPT & 132 & 7 \\
CP & 13 & 9 \\
Total & 794 & 95 \\
\hline
\end{tabular}

Fuente: Elaboración con resultados obtenidos de SPSS versión 24.

Se accedieron a tres cámaras empresariales y dos colegios de profesionistas, dando un total de 95 empleados distribuidos en PYMES por tipo de actividad empresarial o giro, (ver tabla 5).

\section{Tabla 5}

Distribución por giro de la muestra de PYMES

\begin{tabular}{lcc}
\hline \multicolumn{1}{c}{ Giro } & Frecuencia & Porcentaje \\
\hline Transporte & 3 & 1.5 \\
Educativo & 15 & 7.7 \\
Comercial & 12 & 6.2 \\
Construcción & 13 & 6.7 \\
Industrial & 6 & 3.1 \\
Manufacturero & 0 & - \\
Servicio & 36 & 18.5 \\
Otro & 10 & 5.1 \\
Total & 95 & $100 \%$ \\
\hline
\end{tabular}

Fuente: Elaboración con resultados obtenidos de SPSS versión 24. 
LA TRANSFERENCIA DEL MANDO EN LAS PEQUEÑAS Y MEDIANAS EMPRESAS FAMILIARES

La distribución de la muestra de PYMES, por roles de las personas que dieron respuestas al instrumento corresponde a:

Tabla 6

Roles de las personas dentro de muestra de PYMES

\begin{tabular}{lcc}
\hline Roles en la & Frecuencia & Porcentaje \\
Organización & 38 & 19.5 \\
\hline Operativo & 21 & 10.8 \\
Mando Medio & 36 & 18.5 \\
Mando Superior & 95 & $100 \%$ \\
Total & & \\
\hline
\end{tabular}

Fuente: Elaboración con resultados obtenidos de SPSS versión 24.

\section{RESULTADOS Y DISCUSIÓN}

En la tabla 7, se muestran los valores de fiabilidad, basados en el Alfa de Cronbach, el KMO como indicador de adecuación muestral y la Varianza Explicada para cada una de las variables del estudio (Hair, Anderson, Tatham y Black, 2010).

En todos los casos se obtienen valores de fiabilidad superiores a 0.85 , denotando que los ítems miden coherentemente una misma característica. La Varianza Explicada, en todas las variables, es superior a 0.71 revelando que los ítems comparten varianza suficiente, al grado de explicar la varianza total en más del $71 \%$ en todos los casos. Con estos resultados podemos concluir que los ítems diseñados miden de manera fiable y con suficiente consistencia interna las variables del estudio. Existe suficiente validez convergente (Hair et al., 2010) de los ítems y sus variables.

La validez discriminante (Hair et al., 2010) fue determinada por cada uno de los componentes, siguiendo el modelo teórico. Los resultados del AFE se muestran en la tabla 7. 
LA TRANSFERENCIA DEL MANDO EN LAS PEQUEÑAS Y MEDIANAS EMPRESAS FAMILIARES

Tabla 7

Alfa de Cronbach, KMO y varianza explicada de los componentes del estudio

\begin{tabular}{lcccc}
\hline Variable Latente & Componentes & Alpha & KMO & Varianza Explicada* $^{*}$ \\
\hline $\begin{array}{l}\text { Premisas de } \\
\text { decisión para la } \\
\text { sucesión }\end{array}$ & Uso del personal & .967 & .868 & $71.08 \%$ \\
& Vías de comunicación & .968 & .850 & $74.8 \%$ \\
& & .923 & $72.74 \%$ \\
\hline
\end{tabular}

Nota: Se refiere a la varianza explicada por las variables e ítems de cada componente, utilizando Máxima Verosimilitud y rotación varimax en un Análisis Factorial Exploratorio en SPSS v.20.

Fuente: Elaboración propia.

Los resultados de AFE para las variables de cada componente se muestran en las tablas 8, 9 y 10. Como se aprecia, las variables de los componentes Uso del Personal y Programas de Decisión, denotan validez discriminante. En el caso de Vías de Comunicación, sus variables presentan escasa validez discriminante, denotando un área de mejora del cuestionario.

\section{Tabla 8}

Matriz de factores rotados. Variable: uso del personal

\begin{tabular}{|c|c|c|c|c|c|c|c|c|}
\hline Ítems & Autoridad & Información & Integración & $\begin{array}{c}\text { Trabajo en } \\
\text { equipo }\end{array}$ & $\begin{array}{c}\text { Habilidad y } \\
\text { talentos }\end{array}$ & Influencia & Entorno & $\begin{array}{c}\text { Motivación } \\
\text { personal }\end{array}$ \\
\hline P19 & .832 & & & & & & & \\
\hline P17 & .785 & & & & & & & \\
\hline P16 & .765 & & & & & & & \\
\hline P18 & .752 & & & & & & & \\
\hline P21 & .741 & & & & & & & \\
\hline P20 & .729 & & & & & & & \\
\hline P22 & .551 & & & & & & & \\
\hline P38 & & .794 & & & & & & \\
\hline P37 & & .696 & & & & & & \\
\hline P40 & & .675 & & & & & & \\
\hline P41 & & .672 & & & & & & \\
\hline P39 & & .665 & & & & & & \\
\hline P36 & & .649 & & & & & & \\
\hline P43 & & .574 & & & & & .429 & \\
\hline P44 & & .507 & & & & & .456 & \\
\hline P31 & & & .810 & & & & & \\
\hline P32 & & & .797 & & & & & \\
\hline P33 & & & .770 & & & & & \\
\hline
\end{tabular}


LA TRANSFERENCIA DEL MANDO EN LAS PEQUEÑAS Y MEDIANAS EMPRESAS FAMILIARES

\begin{tabular}{|c|c|c|c|c|c|c|c|c|}
\hline P34 & & .454 & .630 & & & & & \\
\hline P35 & & & .587 & & & & & \\
\hline P13 & & & & .687 & & & & \\
\hline P14 & & & & .660 & & & & \\
\hline P10 & .420 & & & .619 & & & & \\
\hline P15 & & & & .600 & & & & .422 \\
\hline P9 & & & & .542 & & & & \\
\hline P11 & & & & .468 & & & & \\
\hline P73 & & & & & .801 & & & \\
\hline P74 & & & & & .681 & & & \\
\hline P75 & & .407 & & & .646 & & & \\
\hline P72 & & .409 & & & .624 & & & \\
\hline P6 & & & & & & .780 & & \\
\hline P5 & & & & & & .757 & & \\
\hline P8 & & & & & & .544 & & \\
\hline P27 & & & & & & .524 & & \\
\hline P12 & & & & & & .455 & & \\
\hline P30 & & & & & & .409 & & \\
\hline P47 & & & & & & & 674 & \\
\hline P48 & & & & & & & .594 & \\
\hline P45 & & & & & & & .556 & \\
\hline P46 & & & & & & & .496 & \\
\hline P42 & & & & & & & .489. & \\
\hline P1 & & & & & & & & .680 \\
\hline P4 & & & & & & & & .621 \\
\hline P2 & & & & & & & & .605 \\
\hline P3 & & & & & & & & .590 \\
\hline
\end{tabular}

Nota: AFE con Máxima Verosimilitud, rotación Varimax con normalización Kaiser en SPPS Versión. Varianza Explicada del $71.08 \%$, y un KMO de 0.868 .

Fuente: Elaboración propia. 
LA TRANSFERENCIA DEL MANDO EN LAS PEQUEÑAS Y MEDIANAS EMPRESAS FAMILIARES

Tabla 9

Matriz de factores rotados. Variable: programas de decisión

\begin{tabular}{ccc}
\hline Ítems & Planeación & Liderazgo \\
\hline P72 & .933 & \\
P73 & .901 & \\
P53 & .721 & .527 \\
P50 & .534 & .435 \\
P52 & .447 & .777 \\
P51 & & .635 \\
P54 & .514 & .623 \\
P49 & & .526 \\
\hline
\end{tabular}

Nota: Análisis Factorial exploratorio con Máxima Verosimilitud, rotación Varimax en SPPS Versión 20. Varianza Explicada del $74.8 \%$, y un KMO de 0.850 .

Fuente: Elaboración propia.

Tabla 10

Matriz de factores rotados. Variable: vías de comunicación

\begin{tabular}{lccc}
\hline & Liderazgo & Confiabilidad & Microambiente \\
\hline P67 & .914 & & \\
P66 & .908 & & -.345 \\
P56 & .895 & -.379 & \\
P69 & .871 & & \\
P58 & .864 & & \\
P61 & .857 & & \\
P57 & .843 & .338 & \\
P70 & .843 & & \\
P62 & .841 & & \\
P55 & .813 & & \\
P75 & .813 & .355 \\
P74 & .807 & .498 \\
P65 & .785 & & \\
P59 & .771 & .413 \\
P63 & .759 & \\
P64 & .727 & \\
P60 & .727 & & \\
P68 & .671 & & \\
P71 & .392 &
\end{tabular}

Nota: Análisis Factorial Exploratorio con Máxima Verosimilitud, rotación Varimax en SPPS Versión 20. Varianza Explicada del $77.56 \%$, y un KMO de .923.

Fuente: Elaboración propia. 
LA TRANSFERENCIA DEL MANDO EN LAS PEQUEÑAS Y MEDIANAS EMPRESAS FAMILIARES

Tabla 11

Alfa de Cronbach, KMO y varianza explicada de las variables del estudio

\begin{tabular}{|c|c|c|c|c|c|}
\hline Premisas LUHMANN & Variables & Alpha & $\mathrm{KMO}$ & $\begin{array}{l}\text { Varianza } \\
\text { Explicada* }\end{array}$ & Ítems \\
\hline \multirow{4}{*}{ Uso del Personal } & Adaptación del Sucesor & .936 & .889 & $71.24 \%$ & P1 - P22 \\
\hline & Garantías y Capacidad de Influir & .899 & .899 & $69.54 \%$ & P23- P41 \\
\hline & Influencia y Motivación & .919 & .880 & $68.72 \%$ & P42- P48 \\
\hline & Dominio de la Empresa & .952 & .857 & $87.49 \%$ & P72 - P75 \\
\hline Programas de & Formación Previa & .859 & .833 & $61.04 \%$ & P49- P54 \\
\hline Decisión & Planificación & .930 & .905 & $93.44 \%$ & P72-P73 \\
\hline \multirow[t]{2}{*}{ Vías de Comunicación } & $\begin{array}{l}\text { Fases de Crecimiento de la } \\
\text { empresa }\end{array}$ & .964 & .928 & $73.24 \%$ & P55 - P71 \\
\hline & $\begin{array}{l}\text { Coordinación de las partes } \\
\text { Interesadas }\end{array}$ & .905 & .500 & $91.33 \%$ & P74 - P75 \\
\hline
\end{tabular}

Nota: Se refiere a la varianza explicada por los ítems del primer factor, utilizando Máxima Verosimilitud y rotación varimax con normalización Kaiser-Leiman en un Análisis Factorial Exploratorio en SPSS v.20.

Fuente: Elaboración propia.

De manera exploratoria, las variables adaptación del sucesor, garantías y capacidad de influir, influencia y motivación, dominio de la empresa, formación previa, planificación, fases de crecimiento de la empresa y coordinación de las partes interesadas, son estimadas por los ítems desarrollados en el presente estudio de manera fiable. De igual forma, las variables denotan consistencia interna en relación a los componentes referidos en el modelo.

Aunque no se confirma estadísticamente el modelo teórico y su sistema de medida (constructo), ni es propósito del estudio hacerlo, se tiene evidencia a favor de las variables que lo integran y del sistema de indicadores desarrollado. Los ítems diseñados miden de manera fiable y con suficiente consistencia interna las variables del estudio; sin embargo, pueden afinarse aún más el cuestionario en los ítems de las variables donde el estudio exploratorio denotó falta de validez discriminante; confiabilidad y microambiente. 
LA TRANSFERENCIA DEL MANDO EN LAS PEQUEÑAS Y MEDIANAS EMPRESAS FAMILIARES

\section{CONCLUSIONES}

La capacidad de establecer premisas de decisión, asociadas a las posiciones de la alta dirección y, secundariamente, en todos los mandos medios y equipos de planificación o selección y reclutamiento en las PYMES, influye directamente en el éxito o fracaso de los procesos sucesorios y de transferencia del mando. La dirección de las PYMES afronta el problema de adoptar decisiones sobre premisas de decisión, de las cuales apenas se puede saber si influyen y cómo, sobre el decidir en situaciones de decisión, o al menos sobre el comportamiento (Luhmann, 2005, p. 72).

Los primeros acercamientos que se muestran en el presente estudio, basados en las premisas de decisión para la sucesión y los enfoques para la transferencia del mando, permiten el abordaje de la problemática de determinar qué decisiones, en lo relativo a la transferencia del mando, deben ser adoptadas por las PYMES para evitar la incertidumbre y el fracaso asociado con la prueba y el error, haciendo manejable la carga de información requerida para tomar la decisión de la transferencia de mando, disminuyendo el riesgo de mortalidad y asegurando la continuidad.

No se trata de decidir basados en la elección entre varias alternativas, ni de tomar una acción basado en algún enfoque racional. Se trata de la comunicación de decisiones previas, hacia el interior de la organización, en respuesta a condiciones inciertas e información contextual que supera por mucho las capacidades de procesamiento de la organización, circunscritas a las premisas de decisión; uso del personal, programas de decisión y vías de comunicación. En este sentido se da respuesta a la pregunta central del estudio. Además, se exploraron las variables asociadas a cada premisa, evidenciando la existencia de las mismas y dando luz sobre las variables (información) que las explican: adaptación del sucesor, garantías y capacidad de influir, influencia y motivación, dominio de la empresa, formación previa, planificación, fases de crecimiento de la empresa y coordinación de las partes interesadas.

El modelo teórico creado para el problema de la transferencia del mando en las PYMES, basado en la teoría de la decisión de Niklas Luhmann y los enfoques racionales para la decisión de la sucesión, se ha operacionalizado y de manera exploratoria, sus variables e indicadores resultan válidos, consistentes y fiables para realizar estudios confirmatorios de mayor alcance. 
LA TRANSFERENCIA DEL MANDO EN LAS PEQUEÑAS Y MEDIANAS EMPRESAS FAMILIARES

No obstante, y al tratarse de un primer acercamiento a esta problemática desde una perspectiva constructivista, se deben tomar los resultados y el modelo propuesto como una aproximación pertinente, pero aún perfectible.

\section{REFERENCIAS BIBLIOGRÁFICAS}

Ackoff, R. (1971). Toward a system of system concepts. Managemente Science, pp. 661-671.

Araya, L. (2012). El proceso de sucesión en la empresa familiar y su impacto en la organización. TEC Empresarial, pp. 29-39.

Bonome, M. (2009). La racionalidad en la toma de decisiones: Análisis de la teoría de la decisión de Herbert A. Simon. La Coruña: Netbiblo, S.L.

Coffman, B. (2014). The family business succession model: an exploratory analysis of factors impacting family business succession. Manhatthan, Kansas.

Colquitt, J. (2011). Justice, trust and trustworthiness: A longitudinal analysis integrating three theorical perspectives. Academy of Management Journal, pp. 1183-1206.

Danco, L. (1975). Beyond survival: A business owner's guide for success. En E. Leon A, Beyond Survival: A Business Owner's Guide for Success. Virginia: Inc., Reston.

Domínguez, C. (2011). Factores psicosociales de éxito en la sucesión de la empresa familiar. Barcelona. Universidad Politécnica de Catalunya.

Eriksson, D. (1997). A Principal exposition of Jean-Lois Le Moignes Systemic Theory. Cybernetics and Human Knowing, p. 41.

Espinosa, L. (2016). ¿Cómo se toman las decisiones organizacionales? Una revisión clásica. Sociológica, pp. 43-78.

Goana, E. (2015). Factores que amenazan la sobrevivencia y continuidad de la empresa familiar en México. Revista Global de Negocios, pp. 17-27.

Hair, J., Anderson, R., Tatham, R. y Black, W. (2010). Análisis Multivariante. Madrid: Prentice Hall Iberia. 
LA TRANSFERENCIA DEL MANDO EN LAS PEQUEÑAS Y MEDIANAS EMPRESAS FAMILIARES

Hania, M. (2012). Factors influencing family business succession. Case study: Gaza family businesses. Islamic University - Gaza.

Hollander, B. (1988). Family-owned businesses: an emerging field or inquiry. Family business review, pp. 145-164.

Leon, M. (2010). Causas de los problemas de sucesión en empresas familiares. flacsoandes.edu.ec, pp. 12-23.

Luhmann, N. (2005). Organización y decisión. Autopoiesis, acción y entendimiento comunicativo. México D.F.: Universidad Iberoamericana-Anthropos.

Luhmann, N. (2010). Organización y decisión. México D.F.: Universidad Iberoamericana-Herder.

Navarrete, J. (2009). Transición generacional: Reto actual de las empresas familiares. Facultad de Contaduria y Administración, UNAM Publicaciones, pp. 60-66.

Pantoja, E. (2016). La sucesión en empresa familiar: una aproximación al estado del arte. UNIMAR: Universidad Mariana, San José de Pasto, Nariño, Colombia, pp. 219-229.

Poza, E. (2011). Empresas familiares. México: Cengage Learning Editores, S.A. de C.V.

Sánchez, E. (2016). Los recursos humanos bajo el enfoque de la teoría de recursos y capacidades. Revista de la Facultad de Ciencias Económicas: Investigación y reflexión, pp. 133-146.

Schumacher, A. (2015). Éxito en la sucesión de empresas familiares. Factores de influencia. España: publicaciones.unirioja.es.

Simon, H. (1979). Rational decision making in business organizatiions. The American Economic Review, pp. 493-513.

Simon, H. (1962). The arquitecture of complexity. Proceeding of the american philosophical society, pp. 467-482. 